\title{
Orientation-specific effects in picture matching and naming
}

\author{
JANICE E. MURRAY \\ University of Otago, Dunedin, New Zealand
}

\begin{abstract}
In two experiments, subjects made timed decisions about the second of two sequentially presented rotated drawings of objects. When the two objects were physically identical, response times to decide whether the two drawings depicted the same object varied as a function of the shortest distance between the orientation of the second drawing and either the orientation of the previous drawing or the upright. This was found for both short $(250-\mathrm{msec})$ and long (2-sec) interstimulus-intervals. The result was also obtained when subjects named the second drawing after deciding whether the first drawing faced left or right. Following repeated experience with the drawings in the left/right task over four blocks of trials, time to name the second drawing in the same-object sequences was independent of orientation. These results suggest that, initially, object- and orientation-specific representations can be formed following a single presentation of a rotated object and subsequently used to identify drawings of the same object at either the same or different orientations. Alignment of the second drawing with either the canonical representation or the new representation at the previous orientation is achieved by normalization through the shortest path. Following experience with the objects, orientation-invariant representations are formed.
\end{abstract}

Successful object recognition occurs despite the fact that changes in an object's orientation, size, and position in space provide the recognition system with markedly different retinal images. Efforts to formulate an account of object constancy have often focused on the effects of changes in orientation on object recognition. The results of many studies have highlighted the adaptability of the human recognition system and have suggested that the form of representation used to recognize objects may vary according to a number of factors. Initially, the time to name line drawings of objects rotated in the image plane increases linearly as a function of the degree of rotation of the object from the upright (see, e.g., Jolicoeur, 1985, 1990; Maki, 1986; McMullen \& Jolicoeur, 1992; Murray, 1995a, $1995 \mathrm{~b}, 1995 \mathrm{c}$ ) for drawings rotated from $0^{\circ}$ to $\pm 120^{\circ}$. This has been interpreted as evidence for orientation-specific object recognition. Objects are represented in permanent memory in terms of upright, internal object axes within a retinally aligned frame of reference (McMullen \& Jolicoeur, 1992). When an object occurs in any orientation other than upright, the input representation of the rotated object must be brought into alignment with the stored

This research was supported by a grant from the Division of Sciences, University of Otago. Some of the data were collected while I was on leave at Dalhousie University, and I thank the Psychology Department for providing me with facilities during my time there. I am grateful to Breton Caplan and David La Rooy for assistance in collecting the data. I thank Pierre Jolicoeur, David La Rooy, Ruth Maki, and an anonymous reviewer for comments on an early version of the manuscript. Correspondence concerning this article can be sent to J. E. Murray, Department of Psychology, University of Otago, Box 56, Dunedin, New Zealand (e-mail: jmur@psy.otago.ac.nz). representation, in order for recognition to occur. The nature of this normalization process is not completely understood, but one candidate process is that of mental rotation (Jolicoeur, 1985, 1988, 1990).

The magnitude of the initial robust orientation effect is significantly reduced after several blocks of naming the same objects (see, e.g., Jolicoeur, 1985; Maki, 1986; McMullen \& Jolicoeur, 1992; Murray, 1995a). This reduced orientation effect following practice does not transfer to a new set of objects (Jolicoeur, 1985; McMullen \& Jolicoeur, 1992; Murray, 1995a), suggesting that individuals learn something specific about these objects. One possibility is that individuals may learn which orientation-invariant parts or attributes reliably distinguish any particular object from all others in the experienced set (Jolicoeur, 1990; Jolicoeur, Snow, \& Murray, 1987). If orientation-invariant information is encoded and used to form new orientationspecific representations, the practice effect should extend to orientations not experienced in the initial blocks of repeated naming, as has been found (Murray, Jolicoeur, McMullen, \& Ingleton, 1993).

Little is known about the origins of the new representations that develop to counter the effects of orientation. Although the initial effects of orientation are robust and generally are reduced gradually after several repeated blocks of trials (Jolicoeur, 1985; Tarr \& Pinker, 1989), diminished effects of orientation have been found after a single repetition of a block of trials (Murray et al., 1993). This suggests that a single viewing of a drawing of a rotated object may be sufficient to trigger the formation of a new representation to accommodate a noncanonical viewthe processing system may begin to encode orientationinvariant information immediately. One difficulty with 
this notion is that learning which orientation-invariant parts uniquely specify each object would seem to require some experience with all the objects in the set. Accordingly, orientation-invariant representations might be expected to develop gradually over repeated viewings.

Work by Tarr and Pinker (1989) suggests that other forms of representation may be possible. Tarr and Pinker measured naming time for three rotated novel shapes that were created to be visually similar and highly confusable. After extensive practice naming the novel shapes, time to name the shapes at new orientations varied as a function of the distance to the nearest learned orientation. Similar results supporting a multiple-views-plus-transformation theory have been obtained with three-dimensional novel objects rotated in depth (Tarr, 1995). This suggests that when orientation-invariant information is not present or is difficult to isolate, multiple orientation-specific representations are formed at the noncanonical orientation. Possibly for all stimuli, the initial response of the processing system may be to form orientation-specific representations of the objects at the experienced orientations. These representations could be formed with a single exposure and would immediately reduce the amount of time-consuming normalization required for recognition of objects at any orientation. The effects of orientation would be further reduced through encoding of orientationinvariant information, if such information were present in the stimuli. Whether orientation-invariant information is immediately encoded or whether an orientation-specific representation is initially formed at the noncanonical orientation is not known. The present experiments investigated the form that these early-stage representations take in picture-matching and naming tasks.

In a picture-matching task, two drawings of objects are presented in sequence, and a subject must decide whether the two drawings depict the same object, ignoring any differences along irrelevant dimensions, such as orientation. Early work (Bartram, 1976; Ellis \& Allport, 1986; Ellis, Allport, Humphreys, \& Collis, 1989) explored the effects of changes in second-picture identity and viewpoint on picture matching. These early studies suggested that recognition of identical objects presented at the same orientation is achieved by transient viewpoint-specific representations, whereas recognition of depth-rotated objects is achieved by means of long-term representations that are viewpoint invariant. More recently, Lawson and Humphreys (1996) have provided evidence to suggest, in contrast, that there are systematic effects of viewpoint change on the recognition of objects rotated in the depth plane. For different-view matches, they found response times were generally faster for pictures that were separated by a smaller normalization in depth, although this viewsimilarity effect did not follow a pattern predicted by a rotation strategy. Lawson and Humphreys argued that both the identical-view advantage and the view-similarity effect could be accounted for if a number of viewpoint-specific representations were stored in memory. Activation of these multiple representations may depend on the degree of similarity between the first and the second pictures, with representations similar in view being activated prior to those less similar in view.

Humphrey and Lupker (1993) also found evidence for viewpoint-specific recognition in a study of objects rotated in the image plane. Subjects performed the picturematching task when the line drawings were presented at $0^{\circ}$ and $120^{\circ}$ in one experiment and at $0^{\circ}$ and $60^{\circ}$ in a second. A response time advantage for pictures presented at the same orientation was found, even over long interstimulusintervals (ISIs) of $2 \mathrm{sec}$. Closer inspection of the results shows this advantage to be true only when the first picture was presented at $0^{\circ}\left(\right.$ e.g., $0^{\circ}-0^{\circ}$ was faster than $0^{\circ}-120^{\circ}$, and not when the first picture was presented at the nonupright orientation of $60^{\circ}$ or $120^{\circ}$ (e.g., $120^{\circ}-120^{\circ}$ was not different from $120^{\circ}-0^{\circ}$ ). This result is interesting because it suggests that access to long-term representations of objects stored in the upright, canonical format can be used to make the match decisions, in addition to information derived solely from the image. Support for this argument is found also in the results of Lawson and Humphreys (1996).

The results of these studies examining the effects of changes of viewpoint in sequential picture-matching tasks provide some evidence that recognition is mediated by relatively durable representations that are viewpoint specific (Humphrey \& Lupker, 1993; Lawson \& Humphreys, 1996). None of the previous studies, however, directly addresses the question of the origins of the representations that are formed at noncanonical orientations. To do so, a number of criteria must be met. First, any one object must occur in only one matching sequence and should not be repeated numerous times during the course of the experiment. This criterion is of obvious necessity if one is to investigate the nature of the initial representations formed at noncanonical orientations, not the representations formed after considerable experience with the objects. None of the experiments discussed above meets this criterion. Second, the objects must be presented at orientations not likely to have been experienced outside the experimental context. Clearly, if either picture in the sequence is presented at a canonical orientation, the match can be made on the basis of a preexisting representation, as is suggested by the results of Humphrey and Lupker (1993). In either case, one cannot determine the role that a newly formed representation might play in performing the matching task. Use of pictures of upright objects rotated in depth about the vertical axis seems to present some difficulties under this criterion. Many of the depthrotated views may be highly familiar prior to entering the experimental situation. One view may constitute the most preferred or canonical view, but it is possible that other, less familiar, upright, depth-rotated views may also be stored in memory through everyday experience. In addition, use of depth-rotated pictures can produce highly similar views that make it difficult to determine what processes are used in deciding whether the two sequential pictures match or not (Humphrey \& Lupker, 1993). In this 
regard, rotation of line drawings in the picture plane may represent a better choice of transformation. Finally, a number of second-picture orientations must be tested in relation to the orientation of the first picture in order to explore fully any potential normalization processes that may operate in the matching process.

The two experiments reported here were designed to meet the criteria described above. In Experiment 1, the effect of rotation in the picture plane on matching sequentially presented pictures was investigated. This experiment allowed an assessment of the representations and processes involved in matching two identical pictures presented at the same or at different orientations. The relative durability of any newly formed representations was assessed by varying ISI. In Experiment 2, the generality of the findings found in the matching task was tested in an identification task.

\section{EXPERIMENT 1}

In Experiment 1, two drawings of familiar objects were presented at nonupright orientations for matching. The first picture in the sequence was always presented at $60^{\circ}$ of clockwise (i.e., $60^{\circ}$ ) or counterclockwise (i.e., $300^{\circ}$ ) rotation in the picture plane. The second picture was presented at one of three orientations: the same orientation as that of the first picture, $120^{\circ}$ from upright, or $-120^{\circ}$ from upright (the negative sign indicates that rotation is in a direction opposite to that of the first picture). These particular orientations allowed for very clear predictions, as will be detailed in the possible outcomes described below.

For the moment, consider trials in which the two pictures match. Depending on the form of representation used to recognize the second picture and enable the match decision, at least four possible outcomes can be described. One possibility is that the match decision is made on the basis of an orientation-specific representation formed following presentation of the first picture. This new representation could then be used in matching the second picture at all the other orientations. If the newly formed representation is used to the exclusion of the canonical representation, time to make the match decision should increase as a function of the distance of the second picture from the first. For sequences in which the first picture was presented at $60^{\circ}$, response times should be fastest for pictures presented at $60^{\circ}\left(0^{\circ}\right.$ from previous orientation $)$, slower for pictures presented at $120^{\circ}\left(60^{\circ}\right.$ from previous orientation), and slowest for pictures presented at $-120^{\circ}$ ( $180^{\circ}$ from previous orientation). This outcome would predict a quadratic accelerating function and is shown in Figure 1 as "Rotation to Previous."

A second possibility is that the orientation-specific representation formed at the presentation of the first picture is used in conjunction with the canonical representation. The second picture could then be recognized by normalizing an image of the object to either the stored representation at $0^{\circ}$ or the new representation $60^{\circ}$ from upright. The work of Tarr and Pinker (1989) suggests that the shortest route between the second picture and either of the two orientation-specific representations would dictate which representation was used to recognize the second picture. For sequences in which the first picture was presented at $60^{\circ}$, response times should be fastest for pictures presented second at $60^{\circ}\left(0^{\circ}\right.$ from nearest orientation at $\left.60^{\circ}\right)$, slower for pictures presented at $120^{\circ}\left(60^{\circ}\right.$ from nearest orientation at $\left.60^{\circ}\right)$, and slowest for pictures presented at $-120^{\circ}\left(120^{\circ}\right.$ from nearest orientation at $\left.0^{\circ}\right)$. This predicted linear function is plotted in Figure 1 as "Rotation to Nearest."

A third outcome rests on the formation of orientationinvariant representations. If orientation-invariant information can be encoded and used to form new representations with a single exposure, this information could be used to detect a match with the second picture. Recognition of the second picture would be independent of orientation, and response times would be equivalent across all second-picture orientations. This prediction is plotted as "No Rotation" in Figure 1.

Finally, a single presentation may be insufficient to form a new representation. Access to canonical representations by means of rotation to the upright would be required in order for the second picture to be recognized and the match decision to be made. Response time would be predicted by distance to the upright, with faster response times at $60^{\circ}$ than at $\pm 120^{\circ}$. This quadratic decelerating function is plotted in Figure 1 as "Rotation to Upright."

If the match/mismatch decision requires access to orientation-specific representations that enable identification of the second object, any new representations formed through presentation of the first picture will be useful only when the two pictures depict the same object. On trials in which the two pictures are different, identification of the second picture can only be achieved through normalization of an image of the second picture to the upright for comparison with canonical representations. Thus, if the representations formed at the experienced orientation are specific to both the object and the orientation, very different patterns of response time effects will be exhibited for match and mismatch trials. Alternatively, if match/mismatch decisions can be made on the basis of matching of visual information without specific knowledge of object identity, a rotation-to-nearest-orientation strategy would be as effective for mismatch trials as for match trials.

Past work has suggested that representations responsible for viewpoint-specific effects may be reduced or eliminated with longer ISIs (Ellis \& Allport, 1986; Ellis et al., 1989), although not always (Humphrey \& Lupker, 1993; Lawson \& Humphreys, 1996). Ellis et al. found, in a no-mask condition, that the advantage for identical-view over different-view matches at ISls of 100 or $500 \mathrm{msec}$ was lost with a longer ISI of $2 \mathrm{sec}$. The effects of ISI have implications for characterizations of the representations as transient, low-level descriptions or as more durable, relatively abstract descriptions. If the representations are of 


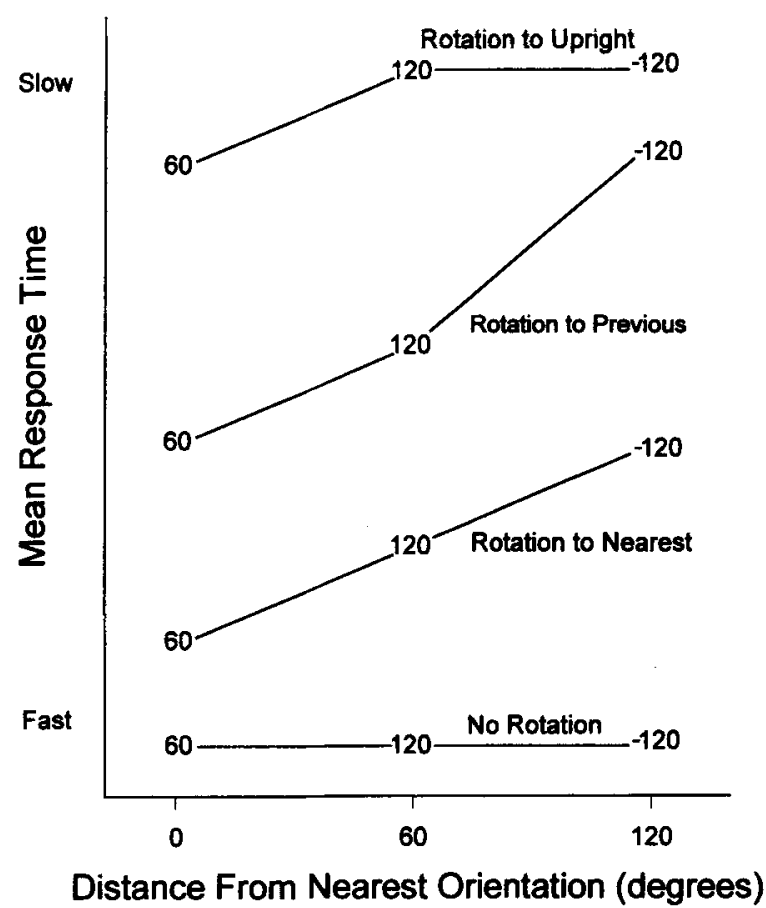

Figure 1. Predicted effects of orientation on response time, plotted as a function of distance from the nearest orientation. Distance from the upright is indicated on the graph by the number plotted at the coordinates of each hypothetical data point. Placement of the functions relative to one another is somewhat arbitrary.

the former type, any effect of orientation, such as those described above, should be observed when the ISI is short, but not when it is relatively long. Alternatively, if the representations are longer lasting, any observed orientationspecific effects should be maintained under both ISI conditions. On the basis of the findings of Ellis (Ellis \& Allport, 1986; Ellis et al., 1989), ISIs of $250 \mathrm{msec}$ and $2 \mathrm{sec}$ were used in the present study.

\section{Method}

Subjects. The subjects were 48 undergraduate students ( 31 females) from the University of Otago and Dalhousie University. All had normal or corrected-to-normal vision and ranged in age from 17 to 40 years. No subject had any previous experience with the stimuli. Each individual participated in a single session of approximately $15 \mathrm{~min}$.

Stimuli and Apparatus. The stimuli were 72 line drawings of animals and objects taken from Snodgrass and Vanderwart (1980). The names of the drawings are listed in the Appendix. All the drawings depicted objects with distinct tops and bottoms. An additional 18 stimuli were used in practice trials. Stored images of the drawings were created by first scanning each drawing and then editing the resulting scanned drawing, to produce uniform images for $60^{\circ}$, $120^{\circ}, 240^{\circ}$, and $300^{\circ}$ of clockwise rotation. The drawings were presented on a Zenith 1492 VGA monitor as black line drawings on a light gray background and subtended an average of $3^{\circ}$ of visual angle. Stimulus presentation and collection of responses were controlled by an IBM-compatible computer using Micro Experimental Laboratory software (Schneider, 1988).
Procedure. A trial began with a $750-\mathrm{msec}$ presentation of a small fixation cross in the center of the screen. The screen was then blanked for approximately $500 \mathrm{msec}$. At the end of this interval, the screen was turned on at the top of the refresh cycle, to reveal the first picture. This first picture in the two-picture sequence was displayed for $500 \mathrm{msec}$. Presentation of the second picture followed $250 \mathrm{msec}$ after the offset of the first picture in the short-ISI condition and $2 \mathrm{sec}$ after offset in the long-ISI condition. The subjects were randomly assigned, in equal numbers, to each of the ISI conditions. The second picture was displayed until the subject responded. Each subject was instructed to decide whether the object depicted in the second picture was the same as or different from the first object, ignoring any differences in orientation. For half of the subjects, same responses were made by pressing the " $\mathrm{n}$ " key on the keyboard with the index finger of the left hand, and different responses were made by pressing the " $b$ " key with the index finger of the right hand. The remaining subjects received the opposite assignment of response to key. Timing began with the onset of the second picture and ended with the subject's keyboard response. Response time sampling from the keyboard indicated a range of plus or minus $1 \mathrm{msec}$. Speed and accuracy in responding were emphasized. Each trial concluded with feedback regarding accuracy of response, displayed for $1 \mathrm{sec}$.

Each subject received a single block of 48 trials. On half the trials, the sequentially presented pictures depicted the same object. On the remaining trials, the second picture depicted an object that was different from the first. Pictures in each different pair were from the same semantic category and had a high degree of similarity in terms of overall visual structure (e.g., cow-deer, desk-dresser). Visually similar objects were used on different trials, to avoid the limited case in which discrimination can occur on the basis of simple diagnostic features (e.g., overall shape). The pictures presented in any pair both faced the same direction when viewed upright. There were approximately equal numbers of leftward- and rightwardfacing pairs across conditions. Each same or different pair was presented only once. The first picture in any sequence was presented $60^{\circ}$ from the upright at either $60^{\circ}$ or $300^{\circ}$ of clockwise rotation. The second picture was presented at one of three orientations. For sequences in which the first picture was presented at $60^{\circ}$, the second picture was presented at $60^{\circ}, 120^{\circ}$, or $240^{\circ}$. For sequences that began with a picture at $300^{\circ}$, the second picture was presented at $300^{\circ}, 240^{\circ}$, or $120^{\circ}$. These pairings allowed for a description of the second picture in terms of distance from the nearest orientation$0^{\circ}, 60^{\circ}$, or $120^{\circ}$ - as was described above. To control for any categoryspecific effects, semantic category members were assigned in approximately equal numbers across orientation and same/different trials. In addition, assignment of object drawing to orientation in each same and different group was counterbalanced so that each picture was presented equally often at each orientation across subjects. Trials were randomly ordered. Twelve practice trials began the experiment. The subjects were provided with additional practice if they made more than two errors during a practice block.

\section{Results}

In order to be included in the group of subjects used for statistical analysis, the subjects had to obtain at least $85 \%$ correct responses. Three subjects failed to meet this criterion and were replaced.

On the basis of response time frequency distributions, response latencies less than $200 \mathrm{sec}$ or greater than $1,575 \mathrm{msec}$ were considered outliers and were removed from any data analyses. This resulted in a loss of less than $1 \%$ of the data. The mean response time for correct responses was calculated for each subject within each ISI 


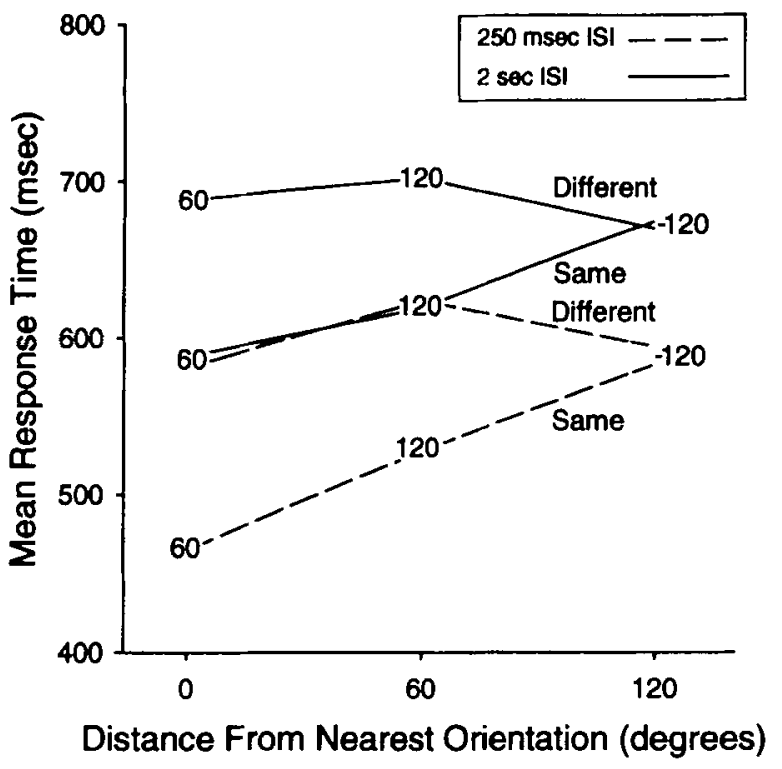

Figure 2. Mean response time as a function of distance from the nearest orientation for same and different trials at short (dashed lines) and long (solid lines) interstimulus intervals (ISIs) in Experiment 1 . Distance from the upright is indicated on the graph by the number plotted at the coordinates of each data point.

condition for same and different trials as a function of distance from the nearest orientation. These mean response times are shown in Figure $2 .^{1}$ All the reported effects were significant at $p<.05$, unless otherwise stated.

Same trials. The mean response times for same responses were submitted to a mixed-model analysis of variance (ANOVA) in which ISI ( $250 \mathrm{msec}$ or $2 \mathrm{sec})$ was a between-subjects variable and distance from nearest orientation $\left(0^{\circ}, 60^{\circ}\right.$, or $\left.120^{\circ}\right)$ was a within-subjects variable. Mean response times were faster in the short-ISI condition $(525 \mathrm{msec})$ than in the long-ISI condition [627 msec; $\left.F(1,46)=12.44, M S_{\mathrm{e}}=29,976\right]$. As is clearly shown in Figure 1, response times increased with increases in distance from the nearest orientation $[F(2,92)=55.37$, $\left.M S_{\mathrm{e}}=2,250\right]$, and this effect of distance from the nearest orientation had a strong linear component $[F(1,46)=$ $\left.117.14, M S_{\mathrm{e}}=2,123\right]$. The residual was not significant $(F<1)$. The orientation effect did not vary across ISI as tested by the ISI $\times$ distance from nearest orientation interaction $\left[F(2,92)=1.72, M S_{\mathrm{e}}=2,250, p>.18\right]$ and the ISI $\times$ distance from nearest orientation ${ }_{\text {linear }}$ interaction $\left[F(1,46)=2.75, M S_{\mathrm{e}}=2,123, p>.10\right]$.

Different trials. Mean response times for different responses were analyzed in the same fashion as those for same responses. As with same responses, the short-ISI condition produced faster response times $(600 \mathrm{msec})$ than did the long-ISI condition $[686 \mathrm{msec} ; F(1,46)=8.19$, $\left.M S_{\mathrm{e}}=32,951\right]$. The effect of distance from nearest orientation was also significant $\left[F(2,92)=7.43, M S_{\mathrm{e}}=1,888\right]$. However, the linear component of this effect was not significant $(F<1)$. There was no difference between the two ISI groups in terms of the overall effect of distance from nearest orientation $\left[F(2,92)=1.95, M S_{\mathrm{e}}=1,888\right.$, $p>.15]$. To further explore the main effect of distance from nearest orientation, planned comparisons first tested the difference between the $60^{\circ}$ and $120^{\circ}$ conditions. A Bonferroni $t$ test indicated that the difference between the means was significant $[t(46)=3.21]$. Mean response times were slower when the second picture was presented $60^{\circ}$ away from the orientation of the first picture than when the second picture was $120^{\circ}$ away from the nearest orientation at $0^{\circ}$. A second comparison, involving $0^{\circ}$ and $60^{\circ}$, also revealed a significant difference $[t(46)=3.67]$. Mean response times were faster when the second picture was presented at the same orientation as the first picture than when it was presented $60^{\circ}$ away from the orientation of the first.

Errors. The error rates are given in Table 1. The only significant effect to arise was one of distance from nearest orientation for same responses $\left[F(2,92)=4.09, M S_{\mathrm{c}}=\right.$ $0.00551]$. There was no evidence of any speed-accuracy tradeoff.

\section{Discussion}

The results reveal a number of clear findings. First, for trials in which the two pictures depicted the same object, response times increased linearly with increases in distance from the nearest of two available orientationseither the orientation of the first picture or the upright. Second, for trials in which the pictures depicted different objects, response times generally varied as a function of distance to the upright. Third, these effects did not change with a long ISI of $2 \mathrm{sec}$.

The results are not consistent with a model that postulates a transient viewpoint-specific representation that mediates identical-view matches and a separate, longterm, viewpoint-invariant representation that is used to make different-view matches (Ellis et al., 1989). Such a model would predict an advantage of identical-view over nonidentical-view matches, with no difference in response times for nonidentical-view matches at different orientations. In addition, the results are inconsistent with one other aspect of the model. The model proposes that viewpointspecific representations used to make identical-view matches are transient and that any identical-view advantage is lost over long ISIs. The viewpoint-specific effects

Table 1

Mean Percent Error Rate for Same and Different Responses at Each Distance From Nearest Orientation in Each Interstimulus Interval (ISI) Condition of Experiment 1

\begin{tabular}{llllc}
\hline & & \multicolumn{3}{c}{ Distance From Nearest Orientation } \\
\cline { 4 - 5 } ISI & Response & $0^{\circ}$ & $60^{\circ}$ & $120^{\circ}$ \\
\hline $250 \mathrm{msec}$ & Same & 3.5 & 6.5 & 5.1 \\
& Different & 3.0 & 5.5 & 5.3 \\
$2 \mathrm{sec}$ & Same & 2.5 & 7.5 & 7.7 \\
& Different & 6.0 & 9.5 & 5.6 \\
\hline
\end{tabular}


observed in this experiment did not disappear with a long ISI of $2 \mathrm{sec}$, and this result is consistent with previous findings (Humphrey \& Lupker, 1993; Lawson \& Humphreys, 1996).

The results do suggest that the initial presentation of a rotated object in an unfamiliar orientation prompts the formation of a new, orientation-specific representation that is available for comparison with the second picture. The decision regarding whether the second picture depicts the same object as the first is made by normalizing the second object through the shortest path to either the new representation or the canonical representation. The systematic, linear nature of the results suggests that the alignment process enacted to allow matching of the image with the representations operates at a constant rate, regardless of whether the representation being accessed is the more familiar canonical representation at upright or the newly formed representation at the previous orientation.

The results for trials in which the first and the second pictures do not match suggest that use of the newly formed representation at the previous orientation is restricted to same-object sequences. The linear effect of distance to the nearest orientation was clearly not found on different trials. Rather, response times were significantly faster for pictures presented at $-120^{\circ}$ than at $120^{\circ}$. The pattern of results on different trials can be accounted for within the rotation-to-upright view, if some interaction occurs between the image of the different picture at $120^{\circ}$ and the representation formed at the previous orientation. A different response for pictures presented at either $120^{\circ}$ or $-120^{\circ}$ would be made following normalization of the image to the upright for comparison with the canonical representation. For pictures presented at $-120^{\circ}$, normalization of the image along this route to upright would be able to proceed without interruption from any newly formed representation. In contrast, for pictures presented at $120^{\circ}$, the first representation encountered through normalization to upright would be the representation of the first picture at $60^{\circ}$. Comparison of the image of the second picture with the representation of the first picture may yield information that signals that the two pictures do not match, but the data clearly suggest that this information is insufficient to allow for a confident different response. Confirmation that the second picture is different from the first could be made after successful identification of the second picture, resulting from normalization of the image to the upright. In this view, the longer response times for $120^{\circ}$ pictures, in comparison with $-120^{\circ}$ pictures, are due to the unsuccessful comparison with the previous orientation representation prior to the necessary comparison with the canonical representation.

The differing pattern of results for different and same trials is important for two reasons. First, they argue against an explanation in terms of rotation of a perceptual frame of reference. It could be argued that the representations used to perform the matching task are indeed orientation invariant. However, the viewpoint-invariant representation of the picture must be described within a reference frame
(Marr \& Nishihara, 1978), and the process of determining the reference frame of the rotated picture may be sensitive to orientation. Once a frame of reference is established for the first picture, this frame of reference could be rotated into alignment with the second picture, resulting in the observed orientation effects. The input pattern could then be described in terms of viewpoint-invariant information, and the match or mismatch determined. Such an argument does not find support in the data. The precise nature of the orientation effect found in the present study depended on whether the two pictures depicted the same or different objects. If any observed effect of orientation on response time is simply the result of establishing a frame of reference, it is not clear why the orientation effect should vary as a function of whether the stimuli to be compared are the same or different.

Second, the contrasting orientation effects for same and different trials also present difficulties for a related viewpoint-invariant explanation of the orientation effect in recognition of objects rotated in the picture plane. Biederman (1987; Biederman \& Gerhardstein, 1993; Hummel \& Biederman, 1992) has argued that orientation effects for picture plane rotation are the consequence of violation of the normal top-bottom and side-of relations among geons, as well as alterations to the vertical, horizontal, and oblique values for attributes of geons. For example, in the present experiment, finding the top of an object rotated $-120^{\circ}$ would likely take longer, relative to an object at $120^{\circ}$, since the object top at $-120^{\circ}$ is further away from the study view. Consistent with this prediction, on match trials, longer RTs were found at $-120^{\circ}$ than at $120^{\circ}$. However, on mismatch trials, the reverse pattern was exhibited; shorter RTs were found at $-120^{\circ}$. An explanation is lacking as to why the consequences of disrupted top-bottom and/or side-of relations brought about by changes in orientation should differ, depending on the match or the mismatch nature of the second picture.

The present findings bear a similarity to those of Koriat and Norman (1988). They found evidence for the operation of two types of mental rotation processes in a normal/ mirror-reversed task. One type was the typical mental rotation process in which an image of the stimulus is rotated to the upright for comparison with representations in memory. The second process they termed backward alignment. This rotation process aligns an image of the stimulus with a short-term visual trace of the immediately preceding stimulus when all aspects of the shape and format of the successive stimuli are in complete correspondence. In a normal/mirror-reversed task in which the successive stimuli share identity and handedness, both processes operate, and response is determined by the process requiring the shortest rotation route.

The present study suggests that similar normalization processes are in operation when identification of rotated objects is required. My results also provide two additional new observations. First, uprighting and backward alignment may reflect two different types of transformation processes, but the results in this experiment suggest that 
they may not be totally independent. As was discussed above, the process of normalizing to the upright appears to interact with the new representation formed at the previous orientation when that representation is located along the uprighting rotation path. Second, the results demonstrate that the new representations formed at the initial orientation are not transient. Effects of normalization to the nearest orientation were found over a relatively long interval of $2 \mathrm{sec}$. This result has important implications when considering the potential of the new representations to serve as more permanent representations for later identification of rotated objects.

\section{EXPERIMENT 2}

It might be argued that the representations used in Experiment 1 to match identical pictures of objects in different orientations have little to do with the representations used for object recognition per se. Biederman and Cooper (1992) have speculated that different tasks may require access to different systems of representation. They proposed that object recognition, as measured in naming tasks, is dependent solely on representations that are viewpoint invariant. Same/different tasks, they argued, may additionally be influenced by an earlier stage of processing that is sensitive to metrically specific information, and any observed viewpoint-dependent effects could be the consequence of low-level visual matching processes unrelated to entry-level recognition. As Tarr and Bülthoff (1995) have argued, such a position is difficult to defend, since there is no a priori reason to suggest that any single task can lay claim to being the true recognition task. Indeed, there are reasons why naming as a task might be considered a less pure measure of recognition, with its additional requirement of postrecognition access to lexical information. Conceptual arguments aside, there are empirical reasons to suggest that both sequential picture matching and naming reflect the operation of viewpointdependent object representations. First, many studies, as was described above, have found orientation effects in naming tasks. Second, comparable orientation effects for both naming and explicit recognition tasks have been found in a number of studies. Lawson has found similar viewpoint-dependent effects in a picture-matching task (Lawson \& Humphreys, 1996) and in a naming task (Lawson, 1994), using the same stimulus set. Koriat, Norman, and Kimchi (1991) have also found similar orientationspecific effects of backward alignment in a task requiring letter classification and one requiring same/different judgments for pairs of letters presented simultaneously.

Experiment 2 was designed to extend the present results obtained with matching to a task requiring naming. The stimulus set and sequences used in Experiment 1 were again used. Upon presentation of the first picture, the subjects decided whether the object would face left or right when viewed upright. When the second picture was presented $500 \mathrm{msec}$ after the left-right response was made, the subjects named the object as quickly as possi- ble. The choice of a nonnaming task for the first picture was intentional. The use of two different responses (i.e., left/right and naming) removes the possibility of a simple response-priming account of any effect of the previous picture on naming time for the second picture. The specific choice of the left/right decision task was motivated by past work suggesting that the representations used in making a left/right decision about objects are also those used in naming objects (Jolicoeur, 1985, 1990). This conclusion is derived from the observation that repeated practice making left/right judgments over several blocks of trials results in a reduced orientation effect for naming the same objects in a later identification task. The possibility that the visual representations are shared across naming and left/right tasks can be further assessed within the present experimental context.

Experiment 2 also explored the relationship between any orientation-specific representations formed initially and the orientation-invariant representations that develop after experience with the objects. Following the initial block of sequential picture presentations, the subjects in the present experiment performed four additional blocks of left-right judgments only. After that left/right practice, in a final block of trials, the subjects then again carried out the same left/right and naming tasks as those in the first block. As was noted above, practice with the left/right task for a set of objects results in a reduced orientation effect for naming, owing to the development of orientation-invariant representations. These representations may provide a more efficient basis for easy recognition and may override any use of orientation-specific representations. Alternatively, although the process of normalizing to the nearest orientation-specific representation takes time, it may still be a faster option than comparing the second picture with orientation-invariant representations in memory. These possibilities were tested in the final block of trials.

\section{Method}

Subjects. The subjects were 36 undergraduate students $(23 \mathrm{fe}-$ males) from the University of Otago. All had normal or correctedto-normal vision and spoke English as a first language. The ages ranged from 17 to 40 years. No subject had any previous experience with the stimuli. The duration of the single session was approximately $45 \mathrm{~min}$.

Stimuli and Apparatus. Sixty-three of the stimuli used in Experiment 1 were selected for use on the basis of easy judgment of the left- or the right-facing view of the object. The apparatus was the same as that in Experiment 1, with two exceptions. A response box and a microphone connected to the computer were used to detect the subjects' responses.

Procedure. The experiment consisted of six blocks of 42 trials. In the first block, each trial began with a centrally presented fixation cross, which remained in view until the subject pressed a response box key. The screen was then blanked for a 500-msec interval. At the end of this interval, the screen was turned on at the top of the refresh cycle, to reveal the first picture. The picture was displayed for $300 \mathrm{msec}$ and was followed by a blank screen. The subjects were instructed to decide as quickly as possible whether the object depicted in the picture would be facing left or right if viewed upright. Left-facing and right-facing responses were made by press- 


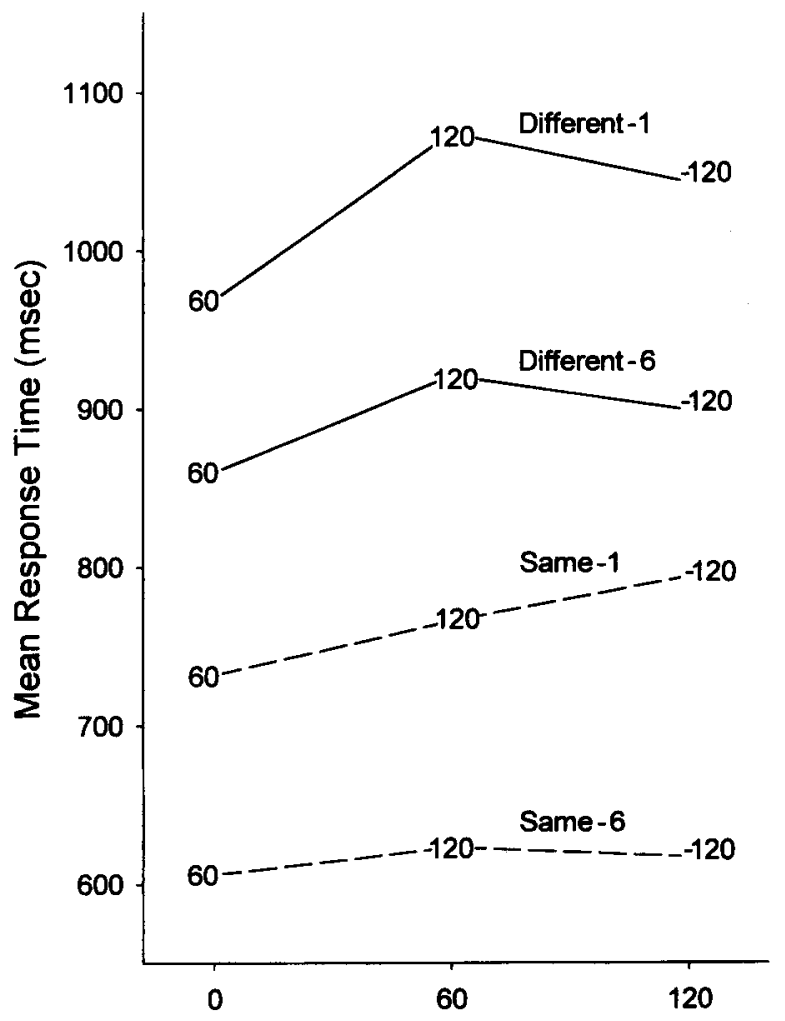

Distance From Nearest Orientation (degrees)

Figure 3. Mean response time as a function of distance from the nearest orientation for same (dashed lines) and different trials (solid lines) in Blocks 1 and 6 of Experiment 2. Distance from the upright is indicated on the graph by the number plotted at the coordinates of each data point.

ing the appropriate marked response box key with the index finger of the left and the right hands, respectively. Presentation of the second picture followed $500 \mathrm{msec}$ after the subject made a response to the first picture. The second picture was displayed until the subject responded by naming aloud the object. Timing began with the onset of the second picture and ended with the subject's verbal naming response. Speed and accuracy in responding were emphasized. At the conclusion of each trial, the subjects were provided with feedback regarding accuracy for the two responses. All other aspects of the block of trials, including orientation of first and second pictures, same/different sequences, and left/right direction, were the same as those described for Experiment 1. Although each object was presented only once in a sequence, across subjects each object drawing appeared equally often at each possible combination of orientation and left/right direction. The first block was preceded by 12 practice trials. The subjects who made more than two errors in the practice block were given an additional 12 trials.

In each of the four blocks of trials that followed Block 1 , only the first picture was presented, and as in Block 1, the subjects were to judge whether the depicted object would face left or right if viewed upright. Across the four blocks, each object was presented once at each of the four possible combinations of left/right direction and $\pm 60^{\circ}$ orientation. Order of presentation of the blocks was randomly assigned.

The final block of trials, Block 6, was identical to Block 1 . The only difference was with respect to assignment of object to orientation and left/right direction. Each object in Block 6 was assigned the opposite orientation to that in Block 1 (i.e., $60^{\circ} / 300^{\circ}$ and $120^{\circ} / 240^{\circ}$ ). In this way, the relationship between the first and the second picture with respect to orientation was identical in the first and the final blocks. At the same time, the objects to be named were presented at an orientation not experienced in the first block. Objects presented facing left in Block 1 were presented facing right in Block 6 , and vice versa. The trial sequence and task in Block 6 were the same as those in Block 1.

\section{Results}

All the subjects reached the accuracy criterion of $85 \%$ correct responses. On the basis of response time frequency distributions, response latencies less than $300 \mathrm{msec}$ and greater than $2,500 \mathrm{msec}$ were considered outliers and were discarded. This criterion was applied for both the left/ right and the naming tasks and resulted in a loss of $1.4 \%$ of the data. Mean response time for correct responses in the left/right task was $810 \mathrm{msec}$ in Block 1 and $648 \mathrm{msec}$ in Block 6 . The corresponding mean percent error associated with Blocks 1 and 6 was 6.4 and 3.4, respectively.

Mean response times for correct responses were calculated for each subject in the naming task for same and different trials at each distance from the nearest orientation for Block 1 and Block 6 . These means are displayed in Figure 3.

Same trials. The mean response times for same responses were analyzed in a repeated measures ANOVA in which block ( 1 vs. 6) and distance from the nearest orientation were the variables. Mean responses were faster in Block $6(616 \mathrm{msec})$ than in Block $1[764 \mathrm{msec}$; $\left.F(1,35)=71.01, M S_{\mathrm{e}}=16,675\right]$. The main effect of distance from the nearest orientation was only marginally significant $\left[F(2,70)=2.92, M S_{\mathrm{e}}=8,299, p<.06\right]$, but the linear component of the orientation effect was significant $\left[F(1,35)=4.90, M S_{\mathrm{e}}=9,401\right]$. The linear component of the distance from the nearest orientation effect interacted significantly with block $[F(1,35)=4.22$, $\left.M S_{\mathrm{e}}=6,224\right]$, indicating that the slope of the effect of distance from the nearest orientation was significantly different across the two blocks. As can be seen in Figure 3, time to name the second picture in Block 1 increased with increases in the distance from the nearest orientation. In contrast, Block 6 performance did not appear to show any effect of distance from the nearest orientation. Separate repeated measures ANOVAs on the data from each block confirmed these observations. There was a significant effect of distance from the nearest orientation in Block $1\left[F(2,70)=3.98, M S_{\mathrm{e}}=8,943\right]$. The linear component of the effect was significant $[F(1,35)=$ $\left.7.46, M S_{\mathrm{e}}=9,515\right]$, with no significant residual $(F<1)$. In Block 6, there was no significant effect of distance from the nearest orientation; both the analysis of the main effect and the test of the linear component revealed that $F<1$.

Different trials. Mean response times for different responses were analyzed in the same fashion as those for same trials. Naming times were faster in Block 6 $(893 \mathrm{msec})$ than in Block $1[1,029 \mathrm{msec} ; F(1,35)=83.68$, $\left.M S_{\mathrm{e}}=11,822\right]$. The effect of distance from the nearest 
Table 2

Mean Percent Error Rate for Naming Responses for Same and Different Trials at Each Distance From Nearest Orientation in the First and Final Blocks of Experiment 2

\begin{tabular}{cllll}
\hline & & \multicolumn{3}{c}{ Distance From Nearest Orientation } \\
\cline { 3 - 5 } Block & Trial Type & $0^{\circ}$ & $60^{\circ}$ & $120^{\circ}$ \\
\hline 1 & Same & 4.2 & 4.7 & 4.6 \\
& Different & 6.5 & 8.4 & 6.5 \\
6 & Same & 0.8 & 1.7 & 1.7 \\
& Different & 3.1 & 2.1 & 6.1 \\
\hline
\end{tabular}

orientation was significant $\left[F(2,70)=11.29, M S_{\mathrm{e}}=\right.$ $11,402]$, as was the linear component of this effect $\left[F(1,35)=14.31, M S_{\mathrm{e}}=8,026\right]$. Test of the residual indicated significant deviation from linearity $[F(1,35)=$ $\left.10.23, M S_{\mathrm{e}}=14,446\right]$. As is evident in Figure 3, orientation effect did not differ significantly across blocks $(F<1)$. The main effect of distance from the nearest orientation was further explored through planned comparisons. First, in the comparison of $60^{\circ}$ and $120^{\circ}$, a Bonferroni $t$ test indicated that there was no significant difference between naming times at $60^{\circ}$ and those at $120^{\circ}$ $[t(34)=1.34]$. The means of these two conditions were collapsed and compared with naming times at $0^{\circ}$. The naming time advantage for objects presented at $0^{\circ}$ from the nearest orientation was significant $[t(34)=4.34]$.

Errors. The error rates for the naming task are given in Table 2. Analyses identical to those for naming times were carried out separately for same and different responses. The only significant effect observed was that of block for same trials $\left[F(1,35)=18.89, M S_{\mathrm{e}}=0.002734\right]$ and for different trials $\left[F(1,35)=6.64, M S_{\mathrm{e}}=0.009542\right]$. In both cases, accuracy improved between the first and the final block.

\section{Discussion}

The main findings are clear and can be summarized as follows. First, time to name the second of two pictures depicting the same object initially increased linearly as a function of distance to the nearest of either the orientation of the previous picture or the upright. Second, when the two pictures depicted different objects, time to name the second varied as a function of distance to the upright. Third, following practice in judging the left/right orientation of the objects, time to name the second of two pictures depicting the same object was independent of orientation.

The results for identical objects in the initial block of naming trials confirm the findings of Experiment 1, observed in a sequential picture-picture matching task, and support the contention that the mechanisms of recognition may not differ in matching and naming tasks. These results are in contrast to the view that, although matching tasks may be influenced by an early stage of processing that is viewpoint dependent, object recognition in naming tasks is based solely on representations that are viewpoint invariant (Biederman \& Cooper, 1992). The present results indicate that when two sequentially presented pictures de- pict the same object, entry-level identification of the second object is achieved by means of multiple viewpointspecific representations that are accessed by normalization processes.

The dependence of naming time on distance to the nearest orientation was found here when the two pictures in the sequence required two different judgments and responses. This eliminates an account based on simple response priming. The effect also reinforces the view that the visual representation of an object is used in common across left/right and naming tasks (Jolicoeur, 1988). Boucart and Humphreys (1997; Boucart, Humphreys, \& Lorenceau 1995), in a different paradigm, have provided evidence to suggest that attention to global form automatically activates identification processes. They had subjects match objects on physical characteristics and found significant semantic-priming effects when the matching task was based on some nonsemantic aspect of global form, such as size, shape, or orientation. The present results are consistent with the findings of Boucart and Humphreys and suggest further that not only does attention to global form (in this case, left/right view of objects) automatically activate identification processes, but it also results in the formation of a new orientation-specific representation.

The different pattern of effects for trials in which the two pictures depicted different objects reinforces the view that the newly formed representations are object specific, as well as orientation specific. In this experiment, the results on different trials conform to the rotation-to-upright view. This is in keeping with the results for different trials in Experiment 1 in showing a pattern consistent with normalization to the upright, but does differ in one respect. As in Experiment 1, response times in Experiment 2 were faster in the $-120^{\circ}$ condition $(971 \mathrm{msec})$ than in the $120^{\circ}$ condition $(997 \mathrm{msec})$. Although the magnitude of the difference $(26 \mathrm{msec}$ ) was comparable with that of Experiment $1(31 \mathrm{msec})$, it was not significant, as was the case in Experiment 1. This lack of significant difference may be a problem of power in the naming task. The naming task necessarily produces more variability in response times, relative to the matching task, owing to the additional requirement of access to lexical information, and as such, naming may not be sensitive enough a measure to detect the effect. Also, the requirements of the two tasks may affect the degree to which the new representation is considered in the process of nomalization to upright. The naming task requires an absolute judgment, whereas the matching task requires a relative judgment. In the latter task, the explicit instruction to decide whether the first and second pictures match may prompt a more thorough and, hence, longer comparison process with the new representation, in an effort to determine a match before normalizing to the upright. In the naming task, where the goal is to identify the object, an unsuccessful comparison with the new representation may result in a relatively immediate continuation of the normalization process to the upright, where the identity of the object can be determined. 
A more definitive account of the processes involved in matching and naming tasks in which the two sequential pictures depict different objects must await further research.

Finally, the results indicate that, following practice, there are no significant effects of orientation in the identification of sequentially presented pictures of the same object. This suggests that once orientation-invariant representations are formed following practice with the set of objects, this representation predominates even when two pictures depicting the same object are presented in sequence. This conclusion rests on the assumption that the practice effect observed with rotated natural objects is indeed the result of the formation of new orientationinvariant representations, rather than of the formation of multiple orientation-specific representations. The work of Murray et al. (1993) provided strong evidence in support of the orientation-invariant account, in showing a reduced orientation effect for objects viewed in a test phase in orientations not experienced in the previous blocks of trials. However, it might be argued that, in the present experiment, although any object in the first block was seen and named at only one orientation, the subject might have adopted a strategy of imagining and forming multiple representations of the same object at the other orientations experienced in the block, in anticipation of viewing the object at these orientations in subsequent trials. This appears unlikely in light of Murray (1995a). In that study, robust effects of orientation were observed when subjects named rotated objects that had been previously seen upright and repeatedly imagined at the test orientations. This indicates that the attenuation of any initial orientation effects with practice cannot be due to imagining and forming representations of objects at a number of specified orientations.

Naming times for different picture sequences continued to show effects of orientation in Block 6, consistent with a strategy of normalization to the upright. Unlike the object in the same picture sequences, objects in the different picture sequences were experienced only during Block 1 and were not seen in Blocks 2-5. Although it is true that a single block of trials can result in an attenuation of the orientation effect (Murray et al., 1993), it has also been shown that several repeated experiences can be required to produce a significant reduction in the magnitude of the orientation effect (e.g., Jolicoeur \& Milliken, 1989). Given the degree of similarity among objects in the present experiment, it might well be expected that determination of the unique feature(s) that would distinguish one object from another in the set would require more than one experience of each object in the set.

\section{GENERAL DISCUSSION}

The aim of the present study was to investigate the origins of long-term representations formed through experience with objects rotated in the picture plane. The find- ings provide some new insights into the processing of rotated objects. First, orientation-specific representations can be formed with a single viewing of a rotated object in an unfamiliar orientation. Second, these new representations, in addition to canonical representations, can be used to match two pictures depicting the same object at either the same or different orientations through an alignment process via the shortest route. Third, the representations last for at least $2 \mathrm{sec}$. Fourth, sensitivity to orientation in the sequential naming task is lost after practice with the rotated objects in a left/right decision task.

An important feature of the present results is the provision of a link between previously reported orientationinvariant effects following practice in naming natural objects (see, e.g., Jolicoeur, 1985; Murray et al., 1993) and the orientation-specific effects found following experience in naming novel shapes (Tarr \& Pinker, 1989). Previous accounts of the practice effect for natural objects have suggested that orientation-invariant representations are responsible for the reduced orientation effects, and in a stimulus set in which orientation-invariant information can usefully discriminate objects, sensitivity to this information is indeed developed (Murray et al., 1993). Only when orientation-invariant information is unavailable or difficult to encode are orientation-specific representations formed at the experienced orientations, as is suggested by the work of Tarr and Pinker. The present results suggest that, regardless of the nature of the stimuli, the initial response of the visual system in countering the effects of orientation may be to form new object- and orientationspecific representations after a single presentation of an object at an unfamiliar orientation. This result with familiar, visually diverse objects provides strong support for the multiple-views hypothesis.

The present results and those of Murray et al. (1993) suggest that, following experience with objects, orientationinvariant representations become the predominant representation when orientation-invariant information is present in the stimulus set and can be isolated. Precisely how much experience is required in order for this transition from orientation-specific to orientation-invariant recognition to take place is yet to be determined. Reduced orientation effects have been found after as little as a single viewing of objects in a block of trials (Murray et al., 1993), but it is not known whether this reduced orientation effect was due to orientation-specific representations or to orientation-invariant representations, since this distinction was not tested until the final block of trials in the Murray et al. study. A second factor to be considered is the effect of intervening items between the first and the second presentations of an object. The processing of an intervening event or events may have some impact on whether the newly formed representation derived from the first presentation endures and is used to identify the second picture. The new representation may not survive the processing of unrelated visual information, prompting development of orientation-invariant repre- 
sentations. These issues relating to the transition from multiple-view to orientation-invariant recognition of natural objects are deserving of further consideration.

The work of Tarr and Pinker (1989) suggests that the rapidly formed multiple views can continue to play an important long-term role in recognition tasks. Support for the notion that the orientation-specific representations may endure and come to serve as more permanent representations is derived from the finding that the effects are present under conditions of both short and long ISIs. This result is in contrast to the findings of Ellis and Allport (1986) and, in part, to the findings of Lawson and Humphreys (1996). Unlike those studies, the present work finds no evidence of transient, viewpoint-specific representation responsible for mediating identical-view matches only. Humphrey and Lupker (1993) also found that the viewpoint-specific representations observable after presentation of a rotated line drawing did not decay rapidly. Any argument suggesting that the initial representations form the basis for more permanent representations used to identify rotated objects would be weakened if the results were obtained at only the short ISI of $250 \mathrm{msec}$. At this interval, the orientation effect could be achieved through matching against an iconic image of the first picture. Although the existence of such a temporary visual trace would not be without interest, evidence of similar orientation-specific effects over a longer interval of $2 \mathrm{sec}$, when any iconic benefit is gone, indicates that the representations formed are potentially long-lasting and likely candidates for the formation of permanent representations later used to identify rotated objects when orientation-invariant information is unavailable.

\section{REFERENCES}

Bartram, D. J. (1976). Levels of coding in picture-picture comparison tasks. Memory \& Cognition, 4, 593-602.

BIEDERMAN, I. (1987). Recognition-by-components: A theory of human image understanding. Psychological Review, 94, 115-147.

BIEDERMAN, I., \& CoOPER, E. E. (1992). Size invariance in visual object priming. Journal of Experimental Psychology: Human Perception \& Performance, 18, 121-133.

Biederman, I., \& Gerhardstein, P. C. (1993). Recognizing depthrotated objects: Evidence and conditions for three-dimensional viewpoint invariance. Journal of Experimental Psychology: Human Perception \& Performance, 19, 1162-1182.

BoucarT, M., \& Humphreys, G. W. (1997). Selection by color and form. Journal of Experimental Psychology: Human Perception \& Performance, 23, 136-153.

Boucart, M., Humphreys, G. W., \& Lorenceau, J. (1995). Automatic access to object identity: Global information, not particular physical dimensions is important. Journal of Experimental Psychology: Human Perception \& Performance, 21, 584-601.

ElLIS, R., \& ALLPORT, D. A. (1986). Multiple levels of representation for visual objects: A behavioural study. In A. G. Cohn \& J. R. Thomas (Eds.), Artificial intelligence and its applications (pp. 245-247). Chichester, U.K.: Wiley.

Ellis, R., Allport, D. A., Humphreys, G. W., \& Collis, J. (1989). Varieties of object constancy. Quarterly Journal of Experimental Psychology, 41A, 775-796.

Hummel, J. E., \& Biederman, I. (1992). Dynamic binding in a neural network for shape recognition. Psychological Review, 99, 480-517.
HumPHREY, G. K., \& LuPKER, S. J. (1993). Codes and operations in picture matching. Psychological Research, 55, 237-247.

Jolicoevr, P. (1985). The time to name disoriented natural objects. Memory \& Cognition, 13, 289-303.

Jolicoeur, P. (1988). Mental rotation and the identification of disoriented objects. Canadian Journal of Psychology, 42, 461-478.

JolicoeuR, P. (1990). Identification of disoriented objects: A dualsystems theory. Mind \& Language, 5, 387-410.

Jolicoeur, P., \& MiLliken, B. (1989). Identification of disoriented objects: Effects of context of prior presentation. Journal of Experimental Psychology: Learning, Memory, \& Cognition, 15, 200-210.

Jolicoeur, P., Snow, D., \& Murray, J. E. (1987). The time to identify disoriented letters: Effects of practice and font. Canadian Journal of Psychology, 41, 303-316.

Koriat, A., \& Norman, J. (1988). Frames and images: Sequential effects in mental rotation. Journal of Experimental Psychology: Learning, Memory, \& Cognition, 14,93-111.

Koriat, A., Norman, J., \& Kimchi, R. (1991). Recognition of rotated letters: Extracting invariance across successive and simultaneous stimuli. Journal of Experimental Psychology: Human Perception \& Performance, 17, 444-457.

LAWSON, R. (1994). The effects of viewpoint on object recognition. Unpublished doctoral thesis, Birmingham University, Birmingham, U.K.

LAWSON, R., \& HumphrEYs, G. W. (1996). Viewpoint-specificity in object processing: Evidence from picture matching. Journal of Experimental Psychology: Human Perception \& Performance, 22, 395-416.

MAKI, R. H. (1986). Naming and location of tops of rotated pictures. Canadian Journal of Psychology, 40, 368-387

MarR, D., \& Nishihara, H. K. (1978). Representation and recognition of the spatial organization of three-dimensional shapes. Proceedings of the Royal Society of London: Series B, 200, 269-294.

McMullen, P. A., \& Jolicoeur, P. (1992). The reference frame and effects of orientation on finding the top of rotated objects. Journal of Experimental Psychology: Human Perception \& Performance, 18, 807-820.

MurRaY, J. E. (1995a). Imagining and naming rotated natural objects. Psychonomic Bulletin \& Review, 2, 239-243.

MurRaY, J. E. (1995b). Negative priming by rotated objects. Psychonomic Bulletin \& Review, 2, 534-537.

Murray, J.E. (1995c). The role of attention in the shift from orientationdependent to orientation-invariant identification of disoriented objects. Memory \& Cognition, 23, 49-58.

Murray, J. E., Jolicoeur, P., McMullen, P. A., \& Ingleton, M. (1993). Orientation-invariant transfer of training in the identification of rotated natural objects. Memory \& Cognition, 21, 604-610.

SCHNEIDER, W. (1988). Micro Experimental Laboratory: An integrated system for IBM PC compatible. Behavior Research Methods, Instruments, \& Computers, 20, 206-217.

SnODGRASS, J. G., \& VANDERWART, M. (1980). A standardized set of 260 pictures: Norms for name agreement, image agreement, familiarity, and visual complexity. Journal of Experimental Psychology: Human Learning \& Memory, 6, 174-215.

TARR, M. J. (1995). Rotating objects to recognize them: A case study of the role of mental transformations in the recognition of three-dimensional objects. Psychonomic Bulletin \& Review, 2, 55-82.

TARR, M. J., \& BüLThOFF, H. H. (1995). Is human object recognition better described by geon structural descriptions or by multiple views? Comment on Biederman and Gerhardstein (1993). Journal of Experimental Psychology: Human Perception \& Performance, 21, $1494-$ 1505.

TARR, M. J., \& PINKER, S. (1989). Mental rotation and orientationdependence in shape recognition. Cognitive Psychology, 21, 233-282.

\section{NOTE}

1. Initial analyses showed that the starting orientation $\left(60^{\circ}\right.$ or $\left.300^{\circ}\right)$ did not interact with any of the factors of interest in either experiment. Therefore, starting orientation was not considered in any of the reported analyses. The results of both reported experiments were confirmed with median response times where no response time data were excluded. 


\section{APPENDIX}

The names of the objects used in Experiment 1 in same and different trials. Each drawing listed for same trials was presented as both the first and second drawing in the picture-picture sequence. First and second drawings for each different trial are as given.

\begin{tabular}{ll}
\multicolumn{1}{c}{ Same } & \multicolumn{1}{c}{ Different } \\
\hline Donkey & Cat-Monkey \\
Seal & Rhinoceros-Elephant \\
Squirrel & Sheep-Goat \\
Bear & Dog-Fox \\
Camel & Deer-Cow \\
Gorilla & Horse-Lion \\
Mouse & Frog-Snail \\
Rabbit & Tiger-Zebra \\
Spider & Ant-Beetle \\
Fly & Grasshopper-Caterpillar \\
Jumper & Boot-Shoe \\
Shirt & Dress-Coat \\
Chair & Desk-Dresser \\
Rocking Chair & Bed-Couch \\
Lamp & Stove-Fridge \\
Swan & Ostrich-Peacock \\
Penguin & Duck-Chicken \\
Bird & Owl-Eagle \\
Toaster & Frying pan-Pot \\
Pitcher & Watering can-Kettle \\
Church & House--Barn \\
Plane & Bicycle-Motorcycle \\
Helicopter & Truck-Train \\
Wagon & Car-Bus \\
\hline & \\
\hline & \\
\hline
\end{tabular}

(Manuscript received June 20, 1997;

revision accepted for publication November 10, 1998.) 